\title{
A CHARACTERISTIC PROPERTY OF THE SPHERE
}

\author{
GIACOMO SABAN
}

\begin{abstract}
Let $S$ be any connected piece of surface in Euclidean three-space, of class $C^{3}$ and $g_{i j}, l_{i j}$ be the coefficients of the first and second fundamental forms of $S$. If these coefficients satisfy the system of differential equations obtained by interchanging the $g_{i j}$ and $l_{i j}$ having same indices in the MainardiCodazzi equations, $S$ is part of a sphere. Furthermore, if two metrics on $S$ satisfy a similar condition, they are proportional.
\end{abstract}

0. Several characterizations of the sphere, all involving the curvature of the second fundamental form, have appeared in the last decade [1-5]. The characterization obtained here is related to the above, in the sense that it follows from a condition in which the second fundamental form is predominant.

1. Let $S$ be a piece of a regular surface of class $C^{3}$ in three-dimensional Euclidean space. Let $g_{i j}$ and $l_{i j}$ respectively denote the coefficients of the first and second fundamental forms of $S$. Suppose there exists some point of $S$ which is not an umbilic: then in some suitable neighbourhood $U$ of this point there is a net of lines of curvature. Choose these lines as coordinate curves $u^{i}=$ constant, $i=1,2$, on $S$ : then

$$
g_{12}(P)=l_{12}(P)=0
$$

for all $P \in U$.

For any function $F=F\left(u^{1}, u^{2}\right)$, let $\partial_{r} F$ denote the partial derivative of $F$ with respect to $u^{r}$. Then the Theorema Egregium can be expressed by the formula [6, p. 120]

$$
K=-\frac{1}{\sqrt{g_{11} g_{22}}}\left(\partial_{1}\left(\frac{\partial_{1}\left(\sqrt{g_{22}}\right)}{\sqrt{g_{11}}}\right)+\partial_{2}\left(\frac{\partial_{2}\left(\sqrt{g_{11}}\right)}{\sqrt{g_{22}}}\right)\right)
$$

and the coefficients of the second fundamental form satisfy two conditions, namely the Mainardi-Codazzi equations for $S$, which, under the assumptions specified above, are the two differential equations [6, pp. 196-198]

$$
\begin{aligned}
& \partial_{2}\left(l_{11} / \sqrt{g_{11}}\right)-\left(l_{22} / g_{22}\right) \partial_{2}\left(\sqrt{g_{11}}\right)=0, \\
& \partial_{1}\left(l_{22} / \sqrt{g_{22}}\right)-\left(l_{11} / g_{11}\right) \partial_{1}\left(\sqrt{g_{22}}\right)=0 .
\end{aligned}
$$

In the sequel we shall refer to equations (1.2) as first Mainardi-Codazzi equations and shall call second Mainardi-Codazzi equations those obtained from (1.2) by interchanging the $g_{i j}$ 's and the $l_{i j}$ 's having same indices.

It should be noted that for the second Mainardi-Codazzi equations to be defined, both $l_{11}$ and $l_{22}$ have to be strictly positive and hence the second fundamental form of $S$ has to be positive definite: as a consequence the Gauss curvature $K$ is also strictly positive on $S$.

Received by the editors April 27, 1981 and, in revised form, November 3, 1981.

1980 Mathematics Subject Classification. Primary 53A05; Secondary 53C45.

(c) 1982 American Mathematical Society 0002-9939/81/0000-0255/802.50 
2. Proposition. Let $S$ be a connected piece of a regular surface of at least class $C^{3}$ in three-dimensional Euclidean space. If the coefficients of the first and second fundamental forms of $S$ satisfy both the first and the second Mainardi-Codazzi equations, $S$ is a piece of a sphere.

PROOF. We claim that under the conditions of the hypothesis all the points of $S$ are umbilics.

In order to prove this statement, assume that it is not fulfilled: then $S$ has at least one point which is not an umbilic and for some suitable neighbourhood $U$ of this point the assumptions of the previous paragraph hold and the coefficients of the first and second fundamental forms of $S$ satisfy the system

$$
\begin{aligned}
& \partial_{2}\left(l_{11} / \sqrt{g_{11}}\right)-\left(l_{22} / g_{22}\right) \partial_{2}\left(\sqrt{g_{11}}\right)=0, \\
& \partial_{1}\left(l_{22} / \sqrt{g_{22}}\right)-\left(l_{11} / g_{11}\right) \partial_{1}\left(\sqrt{g_{22}}\right)=0, \\
& \partial_{2}\left(g_{11} / \sqrt{l_{11}}\right)-\left(g_{22} / l_{22}\right) \partial_{2}\left(\sqrt{l_{11}}\right)=0, \\
& \partial_{1}\left(g_{22} / \sqrt{l_{22}}\right)-\left(g_{11} / l_{11}\right) \partial_{1}\left(\sqrt{l_{22}}\right)=0 .
\end{aligned}
$$

Eliminating $l_{22} / g_{22}$ and $l_{11} / g_{11}$ respectively between the first and third and between the second and fourth of the above, equations

$$
\begin{aligned}
& \partial_{2}\left(l_{11} / \sqrt{g_{11}}\right) \partial_{2}\left(g_{11} / \sqrt{l_{11}}\right)=\partial_{2}\left(\sqrt{g_{11}}\right) \partial_{2}\left(\sqrt{l_{11}}\right), \\
& \partial_{1}\left(l_{22} / \sqrt{g_{22}}\right) \partial_{1}\left(g_{22} / \sqrt{l_{22}}\right)=\partial_{1}\left(\sqrt{g_{22}}\right) \partial_{1}\left(\sqrt{l_{22}}\right)
\end{aligned}
$$

are obtained. Direct calculation then leads to the equations

$$
\begin{aligned}
& \left(\frac{\partial_{2} g_{11}}{g_{11}}-\frac{\partial_{2} l_{11}}{l_{11}}\right)^{2}=0, \\
& \left(\frac{\partial_{1} g_{22}}{g_{22}}-\frac{\partial_{1} l_{22}}{l_{22}}\right)^{2}=0 .
\end{aligned}
$$

From the latter

$$
\begin{aligned}
& l_{11}\left(u^{1}, u^{2}\right)=p\left(u^{1}\right) g_{11}\left(u^{1}, u^{2}\right), \\
& l_{22}\left(u^{1}, u^{2}\right)=q\left(u^{2}\right) g_{22}\left(u^{1}, u^{2}\right),
\end{aligned}
$$

where $p$ and $q$ are two arbitrary functions which appear in the course of the integration of the system (2.3).

Substituting these values in the first and second equations of the initial system (2.1), the conditions

$$
\begin{aligned}
& \left(p\left(u^{1}\right)-q\left(u^{2}\right)\right) \partial_{2}\left(\sqrt{g_{11}}\right)=0 \\
& \left(p\left(u^{1}\right)-q\left(u^{2}\right)\right) \partial_{1}\left(\sqrt{g_{22}}\right)=0
\end{aligned}
$$

are obtained.

Conditions (2.5) are fulfilled if both $\partial_{2}\left(\sqrt{g_{11}}\right)$ and $\partial_{1}\left(\sqrt{g_{22}}\right)$ are simultaneously zero, but in this case $K$ would also be zero, by (1.1): since this cannot be the case, we must have

$$
p\left(u^{1}\right)=q\left(u^{2}\right)
$$

and this implies $p=q=$ constant, hence, by (2.4),

$$
l_{11} / g_{11}=l_{22} / g_{22},
$$

so that the point under consideration is an umbilic. Thus the assumption that $S$ contains points which are not umbilical is absurd and the proposition is proved. Q.E.D. 
3. The previous result can be applied to a more general situation.

Suppose two distinct metrics, $g$ and $l$, having as coefficients the $g_{i j}$ and $l_{i j}$ respectively, are prescribed on some two dimensional connected manifold $M$. Suppose furthermore that

(i) one of the two metrics, say $g$, has nonzero curvature;

(ii) the $g_{i j}$ satisfy the Mainardi-Codazzi equations with respect to the $l_{i j}$ and the converse is also true.

Reduce both metrics simultaneously to orthogonal form (so that $g_{12}=l_{12}=0$ ): in their new form the metrics still satisfy the two sets of Mainardi-Codazzi equations and all the previous calculations hold. Thus the following proposition is obtained.

Proposition. Let $M$ be a connected 2-disc manifold under two metrics $g, l$, such that the curvature of one of the metrics is nonzero. If both metrics satisfy the Mainardi-Codazzi equations with respect to each other, then $l=C g$, where $C \in \mathbf{R}$ is a constant. ${ }^{1}$

\section{REFERENCES}

1. R. Schneider, Closed convex hypersurfaces with second fundamental form of constant curvature, Proc. Amer. Math. Soc. 35 (1972), 230-233.

2. D. Koutroufiotis, Two characteristic properties of the sphere, Proc. Amer. Math. Soc. 44 (1974), 176-178.

3. U. Simon, Characterizations of the sphere by the curvature of the second fundamental form, Proc. Amer. Math. Soc. 55 (1976), 382-384.

4. T. Koufogiorgos and T. Hasanis, A characteristic property of the sphere, Proc. Amer. Math. Soc. 68 (1977), 303-305.

5. G. Stamou, Global characterizations of the sphere, Proc. Amer. Math. Soc. 68 (1977), 328-330.

6. K. Strubecker, Differentialgeometrie, III, Theorie der Flächenkrümmung, 2nd ed., de Gruyter, Berlin, 1969.

Istituto di MAtematica, Universita Degli Studi Dell' AQUila Degli ABRUZZI, L'AQUILA, ITALY

\footnotetext{
${ }^{1}$ The writer is indebted to Professor Udo Simon of the Technische Universität in Berlin for having suggested the last proposition and both to Professor Simon and to the referee for corrections in the earlier versions of this paper.
} 